\title{
Two Poset Polytopes
}

\author{
Richard P. Stanley* \\ Department of Mathematics, Massachusetts Institute of Technology, Cambridge, MA 02139
}

\begin{abstract}
Two convex polytopes, called the order polytope $\mathcal{O}(P)$ and chain polytope $\mathscr{C}(P)$, are associated with a finite poset $P$. There is a close interplay between the combinatorial structure of $P$ and the geometric structure of $\mathcal{O}(P)$. For instance, the order polynomial $\Omega(P, m)$ of $P$ and Ehrhart polynomial $i(\mathcal{O}(P), m)$ of $\mathcal{O}(P)$ are related by $\Omega(P, m+1)=i(\mathcal{O}(P), m)$. A "transfer map" then allows us to transfer properties of $\mathcal{O}(P)$ to $\mathscr{C}(P)$. In particular, we transfer known inequalities involving linear extensions of $P$ to some new inequalities.
\end{abstract}

\section{The Order Polytope}

Our aim is to investigate two convex polytopes associated with a finite partially ordered set (poset) $P$. The first of these, which we call the "order polytope" and denote by $\mathcal{O}(P)$, has been the subject of considerable scrutiny, both explicit and implicit. Much of what we say about the order polytope will be essentially a review of well-known results, albeit ones scattered throughout the literature, sometimes in a rather obscure form. The second polytope, called the "chain polytope" and denoted $\mathscr{C}(P)$, seems never to have been previously considered per se. It is a special case of the vertex-packing polytope of a graph (see Section 2) but has many special properties not in general valid or meaningful for graphs. There is a surprising connection (Section 3) between $O(P)$ and $\mathscr{C}(P)$ which will allow us to "transfer" properties of $\mathscr{O}(P)$ over to $\mathscr{C}(P)$.

Given the poset $P=\left\{x_{1}, \ldots, x_{n}\right\}$ (where by standard abuse of notation we identify $P$ with its set of points), the set $\mathbb{R}^{P}$ of all functions $f: P \rightarrow \mathbb{R}$ is an $n$-dimensional real vector space with a scalar product defined by $\langle f, g\rangle=$ $\sum_{x \in P} f(x) g(x)$, which makes $\mathbb{R}^{P}$ a Euclidean space. In particular, we can talk about convex subsets of $\mathbb{R}^{P}$ and their volumes, orthogonal projections, etc.

\footnotetext{
*Partially supported by NSF Grant No. 8104855-MCS and by a Guggenheim Fellowship.
} 
Definition 1.1. The order polytope $\mathcal{O}(P)$ of the poset $P$ is the subset of $\mathbb{R}^{P}$ defined by the conditions

$$
\begin{aligned}
0 & \leq f(x) \leq 1, & & \text { for all } x \in P, \\
f(x) & \leq f(y) & & \text { if } x \leq y \text { in } P .
\end{aligned}
$$

Note that $\mathcal{O}(P)$ is a convex polytope since it is defined by linear inequalities and is bounded because of (1). Clearly, because of (2), we can replace (1) by the conditions

$$
\begin{array}{cl}
0 \leq f(x), & \text { if } x \text { is a minimal element of } P, \\
f(x) \leq 1, & \text { if } x \text { is a maximal element of } P .
\end{array}
$$

By the transitivity of $P$, we can replace (2) by the equivalent conditions

$$
f(x) \leq f(y) \quad \text { if } y \text { covers } x \text { in } P .
$$

Let $\sigma: P \rightarrow\{1, \ldots, n\}$ be a linear extension (order-preserving bijection) of $P$. We identify $\sigma$ with the permutation $y_{1}, \ldots, y_{n}$ of the elements $x_{1}, \ldots, x_{n}$ of $P$ defined by $\sigma\left(y_{i}\right)=i$. All functions $f \in \mathbb{R}^{P}$ satisfying $0 \leq f\left(y_{1}\right) \leq \cdots \leq f\left(y_{n}\right) \leq 1$ belong to $\mathcal{O}(P)$. These functions form an $n$-dimensional simplex, so we conclude $\operatorname{dim} \mathcal{O}(P)=n$. It is easily seen that conditions $\left(1^{\prime}\right)$ and $\left(2^{\prime}\right)$ are independent, so they define the facets $[(n-1)$-dimensional faces] of $\mathcal{O}(P)$. More precisely a facet of $\mathcal{O}(P)$ consists of those $f \in \mathcal{O}(P)$ satisfying exactly one of the following conditions:

$$
\begin{array}{ll}
f(x)=0, & \text { for some minimal } x \in P, \\
f(x)=1, & \text { for some maximal } x \in P, \\
f(x)=f(y), & \text { for some } y \text { covering } x \text { in } P .
\end{array}
$$

It is convenient to state the above conditions in a more uniform way. Let $\hat{P}$ be the poset obtained from $P$ by adjoining a minimum element $\hat{0}$ and a maximum element $\hat{1}$. Define a polytope $\hat{\mathcal{O}}(P)$ to be the set of functions $g \in \mathbb{R}^{\hat{P}}$ satisfying

$$
\begin{array}{ll}
g(\hat{0})=0, & g(\hat{1})=1, \\
g(x) \leq g(y) & \text { if } x \leq y \text { in } \hat{P} .
\end{array}
$$

The linear map $\rho: \hat{\mathcal{O}}(P) \rightarrow \mathcal{O}(P)$ obtained by restriction to $P$ is clearly a bijection and hence (since $\rho$ is linear) defines a combinatorial equivalence of polytopes. Thus by (3) a facet of $\mathcal{O}(P)$ consists of those $g \in \mathcal{O}(P)$ satisfying $g(x)=g(y)$ for some fixed pair $(x, y)$ for which $y$ covers $x$ in $\hat{P}$. In particular, the number of facets of $\hat{O}(P)$ or $\hat{\mathcal{O}}(P)$ is the number $c(\hat{P})$ of cover relations in $\hat{P}$, or equivalently $c(P)+a+b$, where $P$ has $a$ minimal elements and $b$ maximal elements.

We now wish to determine the entire facial structure of $\hat{\mathcal{O}}(P)$, or equivalently of $\mathcal{O}(P)$. Since every face is an intersection of facets, it follows that a face $F_{\pi}$ of $\hat{\mathcal{O}}(P)$ corresponds to certain partitions $\pi=\left\{B_{1}, \ldots, B_{k}\right\}$ of $\hat{P}$ into nonempty pairwise disjoint blocks, viz.,

$$
F_{\pi}=\left\{g \in \hat{\mathcal{O}}(P): g \text { is constant on the blocks } B_{i} \text { of } \pi\right\} .
$$




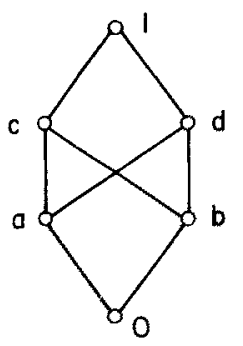

Fig. 1

It remains to determine for which $\pi F_{\pi}$ is a face, and which are the distinct faces $F_{\pi}$. Call $\pi$ a face partition if $F_{n}$ is a face of $\hat{P}$. It is clear that if $\pi$ is a face partition, then $\pi$ is connected, i.e., every block $B$ of $\pi$ is connected as an (induced) subposet of $\hat{\boldsymbol{P}}$. Call a partition $\pi=\left\{B_{1}, \ldots, B_{k}\right\}$ closed if for any $i \neq j$ there is $g \in F_{\pi}$ such that $g\left(B_{i}\right) \neq g\left(B_{j}\right)$. Every partition $\pi$ has a unique coarsening $\bar{\pi}$ for which $\bar{\pi}$ is closed and $F_{\pi}=F_{\bar{\pi}}$. Moreover, if $\pi=\left\{B_{1}, \ldots, B_{k}\right\}$ is a closed face partition then $\operatorname{dim} F_{\pi}=k-2$ [since if $\hat{0} \in B_{3}$ and $\hat{1} \in B_{j}$ then $g \in F_{\pi}$ satisfies $g\left(B_{i}\right)=0$ and $\left.g\left(B_{j}\right)=1\right]$. Hence it remains to describe the closed face partitions. This description was apparently first explicitly observed by Geissinger [6]. We will state Geissinger's result below (Theorem 1.2) but will omit the rather straightforward proof.

Define a binary relation $\leq_{\pi}$ on $\pi$ by setting $B_{1} \leq B_{j}$ if $x \leq y$ for some $x \in B_{i}$ and $y \in B_{j}$. Call $\pi$ compatible if the transitive closure of $\leq_{\pi}$ is a partial order (i.e., is antisymmetric). If $\pi$ is compatible then every block $B$ of $\pi$ is convex; i.e., if $x, z \in B$ and $x<y<z$, then $y \in B$. The converse is false; e.g., let $\hat{P}$ be given by Fig. 1 . The partition into blocks $\hat{0}, a d, b c, \hat{1}$ is connected and convex, but not compatible.

Theorem 1.2. A partition of $P$ is a closed face partition if and only if it is connected and compatible. (In particular, the partition $\pi$ into a single block $\hat{P}$ yields the empty set $F_{\pi}=\varnothing$, which we regard as a face.)

Thus the lattice of faces $\hat{O}(P)$ [or $\hat{\mathcal{O}}(P)]$ is isomorphic to the lattice of connected compatible partitions of $\hat{P}$, ordered by reverse refinement. For instance, if $P=\{a, b\}$ is a two-element antichain, then $\mathcal{O}(P)$ is a square and Fig. 2 depicts its face lattice (with $\hat{0}$ and $\hat{1}$ written 0 and 1 ).

Define a filter (or dual order ideal, up-set, or increasing subset) of $P$ to be a subset $I$ of $P$ such that if $x \in I$ and $y \geq x$, then $y \in I$. Let $\chi_{I}: P \rightarrow \mathbb{R}$ denote the

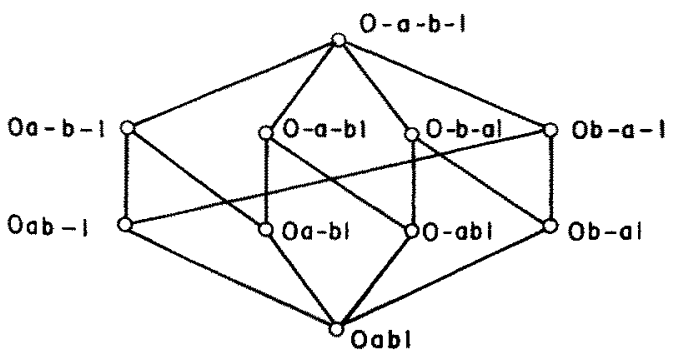

Fig. 2 
characteristic function of $I$; i.e.,

$$
\chi_{J}(x)= \begin{cases}1, & x \in I \\ 0, & x \notin I\end{cases}
$$

The following corollary is immediate from Theorem 1.2 and can also be easily proved directly.

Corollary 1.3. The vertices of $\mathcal{O}(P)$ are the characteristic functions $\chi_{I}$ of filters $I$ of $P$. In particular, the number of vertices of $O(P)$ is the number of filters of $P$.

\section{The Chain Polytope}

Let us define a second polytope associated with a poset $P=\left\{x_{1}, \ldots, x_{n}\right\}$.

Definition 2.1. The chain polytope $\mathscr{C}(P)$ of the poset $P$ is the subset of $\mathbb{R}^{P}$ defined by the conditions

$$
\begin{aligned}
& 0 \leq g(x), \quad \text { for all } x \in P, \\
& g\left(y_{1}\right)+\cdots+g\left(y_{k}\right) \leq 1, \quad \text { for every chain } y_{1}<\cdots<y_{k} \text { of } P .
\end{aligned}
$$

Again it is clear that $\mathscr{C}(P)$ is a convex polytope. Since $\mathscr{C}(P)$ contains the $n$-dimensional simplex $\left\{g \in \mathbb{R}^{P}: g(x) \geq 0\right.$ for all $x \in P$ and $g\left(x_{1}\right)+\cdots+g\left(x_{n}\right)$ $\leq 1\}$, we have $\operatorname{dim} \mathscr{C}(P)=n$. In view of (5) we can replace (6) by

$$
g\left(y_{1}\right)+\cdots+g\left(y_{k}\right) \leq 1, \quad \text { for every maximal chain } y_{1}<\cdots<y_{k} \text { of } P \text {. }
$$

Conditions (5) and $\left(6^{\prime}\right)$ are easily seen to be independent and thus define the facets of $\mathscr{C}(P)$. In particular, the number of facets of $\mathscr{C}(P)$ is equal to $n+m(P)$, where $m(P)$ is the number of maximal chains of $P$.

A description of the faces of $\mathscr{C}(P)$ analogous to Theorem 1.2 seems messy and will not be pursued here. However, we do have a simple description of the vertices analogous to Corollary 1.3. Define an antichain of $P$ to be a subset $A$ of pairwise incomparable elements of $P$.

Theorem 2.2. The vertices of $\mathscr{C}(P)$ are the characteristic functions $\chi_{A}$ of antichains of $P$. In particular, the number of vertices of $\mathscr{C}(P)$ is equal to the number of antichains of $P$.

Proof. Clearly each $\chi_{A} \in \mathscr{C}(P)$. Since $0 \leq g(x) \leq 1$ for all $g \in \mathscr{C}(P)$ and $x \in P$, it follows that $\chi_{A}$ is a vertex of $\mathscr{C}(P)$.

Conversely, suppose $g \in \mathscr{C}(P)$ and $g \neq \chi_{A}$ for any antichain $A$ of $P$. Let $Q=\{x \in P: 0<g(x)<1\}$. Let $Q_{1}$ be the set of minimal elements of $Q$ and $Q_{2}$ the set of minimal elements of $Q-Q_{1}$. One easily sees that since $g \neq \chi_{A}, Q_{1}$ and 
$Q_{2}$ are nonempty. Define

$$
\varepsilon=\min \left\{g(x), 1-g(x): x \in Q_{1} \cup Q_{2}\right\}
$$

Define $g_{1}, g_{2}: P \rightarrow \mathbb{R}$ by

$$
\begin{aligned}
& g_{1}(x)= \begin{cases}g(x), & x \notin Q_{1} \cup Q_{2} \\
g(x)+\varepsilon, & x \in Q_{1} \\
g(x)-\varepsilon, & x \in Q_{2},\end{cases} \\
& g_{2}(x)= \begin{cases}g(x), & x \notin Q_{1} \cup Q_{2} \\
g(x)-\varepsilon, & x \in Q_{1} \\
g(x)+\varepsilon, & x \in Q_{2} .\end{cases}
\end{aligned}
$$

It is clear that $g_{1}, g_{2} \in \mathscr{C}(P)$. Since $g_{1} \neq g_{2}$ and $g=\frac{1}{2}\left(g_{1}+g_{2}\right)$, it follows that $g$ is not a vertex of $\mathscr{C}(P)$.

Theorem 2.2 is already known within a graph-theoretical context. Let $G$ be a graph (with no loops and multiple edges) on a vertex set $V=\left\{x_{1}, \ldots, x_{n}\right\}$. Let $\mathscr{V}(G) \subseteq \mathbb{R}^{V}$ denote the convex hull of the characteristic functions $\chi_{A}$ of independent (stable) sets $A$ of vertices; i.e., no two vertices in $A$ are adjacent in $G$. Then $\mathscr{V}(G)$ is called the vertex-packing polytope of $G$. In particular, given a poset $P$ define its comparability graph $\operatorname{Com}(P)$ to be the graph whose vertices are the elements of $P$, with $x, y \in P$ adjacent if $x<y$ or $y<x$. Then an independent set of vertices of $\operatorname{Com}(P)$ is just an antichain $A$ of $P$, so by Theorem 2.2 we have $\mathscr{V}(\operatorname{Com}(P))=\mathscr{C}(P)$. But since comparability graphs are perfect (e.g., [6, Thm. 5.34]) it follows from [2, Thm. 3.1] (or see [7, Thm. 3.14]) that the facets of $\mathscr{V}(\operatorname{Com}(P))$ are given by $(5)$ and $\left(6^{\prime}\right)$.

There is a well-known bijection between filters $I$ and antichains $A$ of $P$, viz.,

$$
\begin{aligned}
& I=\{y: y \geq x \text { for some } x \in A\}, \\
& A=\text { set of minimal elements of } I .
\end{aligned}
$$

Thus from Corollary 1.3 and Theorem 2.2 it follows that $O(P)$ and $\mathscr{C}(P)$ have the same number of vertices. In general, however, $\mathcal{O}(P)$ and $\mathscr{C}(P)$ need not have the same number of $i$-dimensional faces for $i>0$ (and hence need not be combinatorially equivalent). For instance, if $P$ is given by Fig. 3, then $\mathcal{O}(P)$ has eight facets and $\mathscr{C}(P)$ has nine facets. There is, however, one class of posets for which $\mathcal{O}(P)$ and $\mathscr{C}(P)$ are in fact combinatorially equivalent.

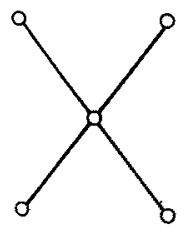

Fig. 3 
Theorem 2.3. Suppose $P$ has length at most one (i.e., $P$ has no three-element chains). Then $\mathcal{O}(P)$ and $\mathscr{b}(P)$ are affinely equivalent and hence combinatorially equivalent.

Proof. Define a nonsingular affine transformation $\zeta: \mathbb{R}^{P} \rightarrow \mathbb{R}^{P}$ by

$$
(\zeta f)(x)= \begin{cases}f(x), & \text { if } x \text { is a minimal element of } P \\ 1-f(x), & \text { otherwise }\end{cases}
$$

It is routine to check that the image of $\mathscr{O}(P)$ under $\zeta$ is $\mathscr{C}(P)$, and the proof follows.

In Section 4 we generalize the fact that for any $P, O(P)$ and $\mathscr{C}(P)$ have the same number of vertices.

\section{A Connection Between $O(P)$ and $\mathscr{C}(P)$}

In this section we construct a map $\phi: \mathcal{O}(P) \rightarrow \mathscr{C}(P)$ with several nice properties. This will allow us to transfer certain properties of $\mathcal{O}(P)$ over to $\mathscr{C}(P)$.

Definition 3.1. Let $P$ be a finite poset, and define the transfer map $\phi: \mathcal{O}(P) \rightarrow$ $\mathscr{C}(P)$ as follows: If $f \in \mathcal{O}(P)$ and $x \in P$ then

$$
(\phi f)(x)=\min \{f(x)-f(y): x \text { covers } y \text { in } P\} .
$$

Theorem 3.2. (a) The transfer map $\phi$ is a continuous, piecewise-linear bijection from $\mathcal{O}(P)$ onto $\mathscr{C}(P)$.

(b) Let $m$ be a positive integer and $f \in \mathcal{O}(P)$. Then $m f(x) \in \mathbb{Z}$ for all $x \in P$ if and only if $m(\phi f)(x) \in \mathbb{Z}$ for all $x \in P$.

Proof. (a) Continuity is immediate from the definition (7). Moreover, for each linear extension $y_{1}, \ldots, y_{n}$ of $P, \phi$ is linear on the simplex defined by $0 \leq f\left(y_{1}\right) \leq$ $\cdots \leq f\left(y_{n}\right) \leq 1$. Since these simplices clearly cover $\mathcal{O}(P)$, it follows that $\phi$ is piecewise-linear. Now define $\psi: \mathscr{C}(P) \rightarrow \mathcal{O}(P)$ by

$$
(\psi g)(x)=\max \left\{g\left(y_{1}\right)+\cdots+g\left(y_{k}\right): y_{1}<\cdots<y_{k}=x\right\} .
$$

One checks that $(\phi \psi) f=f$ and $(\psi \phi) g=g$ for all $f \in \mathcal{O}(P)$ and $g \in \mathscr{C}(P)$. Hence $\phi$ is a bijection (with inverse $\psi$ ).

(b) This result is immediate from (7) and (8).

\section{The Ehrhart Polynomial}

Let $\mathscr{P}$ be a $d$-dimensional convex polytope in $\mathbb{R}^{n}$ with integer vertices. If $m$ is a positive integer then define

$$
i(\mathscr{P}, m)=\operatorname{card}\left(m \mathscr{P} \cap \mathbf{Z}^{n}\right) .
$$


In other words, $i(\mathscr{P}, m)$ is equal to the number of points $\alpha \in \mathscr{P}$ such that $m \alpha \in \mathbb{Z}^{n}$. It is known that $i(\mathscr{P}, m)$ is a polynomial function of $m$ of degree $d$, called the Ehrhart polynomial of $\mathscr{P}$. When $d=n$ the leading coefficient of $i(\mathscr{P}, m)$ is the volume $V(\mathscr{P})$ of $\mathscr{P}$. For these and other facts concerning $i(\mathscr{P}, m)$, see, e.g., [15].

Now let $P$ be a finite $n$-element poset and $m$ a positive integer and define $\Omega(P, m)$ to be the number of order-preserving maps $\eta: P \rightarrow\{1, \ldots, m\}$; i.e., if $x \leq y$ in $P$ then $\eta(x) \leq \eta(y)$. Then $\Omega(P, m)$ is a polynomial function of $m$ of degree $n$, called the order polynomial of $P$. The leading coefficient of $\Omega(P, m)$ is $e(P) / n$ !, where $e(P)$ is the number of linear extensions of $P$. For these and other facts concerning $\Omega(P, m)$, see, e.g., [12] and [13, Sections 13 and 19].

Theorem 4.1. The Ehrhart polynomials of $\mathcal{O}(P)$ and $\mathscr{C}(P)$ are given by

$$
i(\mathcal{O}(P), m)=i(\mathscr{C}(P), m)=\Omega(P, m+1) \text {. }
$$

Proof. By definition, $i(\mathcal{O}(P), m)$ is equal to the number of order-preserving maps $f: P \rightarrow \mathbb{R}$ satisfying $0 \leq f(x) \leq 1$ and $m f(x) \in \mathbb{Z}$ for all $x \in P$. This is equivalent to the condition that $m f: P \rightarrow\{0,1, \ldots, m\}$ is order-preserving, so $i(\mathcal{O}(P), m)=\Omega(P, m+1)$. But Theorem 3.2(b) implies that $i(\mathcal{O}(P), m)=$ $i(\mathscr{C}(P), m)$, and the proof follows.

Since the leading coefficient of $i(\mathscr{P}, m)$ is $V(\mathscr{P})$ (when $\operatorname{dim} \mathscr{P}=n$ and $\left.\mathscr{P} \subset \mathbb{R}^{n}\right)$ and that of $\Omega(P, m+1)$ is $e(P) / n$ !, there follows

Corollary 4.2. The volumes of $\mathcal{O}(P)$ and $\mathscr{C}(P)$ are given by

$$
V(\mathcal{O}(P))=V(\mathscr{C}(P))=e(P) / n !
$$

It would be interesting to find other vertex-packing polytopes whose volumes have a simple combinatorial interpretation. Let us also mention that a method similar to the proof of Corollary 4.2 for showing that two convex polytopes have the same volume appears in [14].

Example 4.3. Let $F_{n}$ denote the $n$-element fence, i.e., the poset with elements $x_{1}, \ldots, x_{n}$ and cover relations

$$
\begin{array}{ll}
x_{1}<x_{i+1}, & \text { if } i \text { is odd, } \\
x_{1}>x_{i+1}, & \text { if } i \text { is even. }
\end{array}
$$

A bijection $\sigma: F_{n} \rightarrow\{1, \ldots, n\}$ is order-preserving if and only if the permutation $\sigma\left(x_{1}\right), \sigma\left(x_{2}\right), \ldots, \sigma\left(x_{n}\right)$ of $\{1, \ldots, n\}$ is alternating, i.e., $\sigma\left(x_{1}\right)<\sigma\left(x_{2}\right)>\sigma\left(x_{3}\right)<$ $\cdots$. Hence $e\left(F_{n}\right)$ is the number $E_{n}$ of alternating permutations of $\{1, \ldots, n\} . E_{n}$ is an Euler number and is well-known (e.g., [3, pp. 258-259]) to satisfy

$$
\sum_{n \geq 0} \frac{E_{n} x^{n}}{n !}=\sec x+\tan x
$$


The chain polytope $\mathscr{C}\left(F_{n}\right)$ may be identified with the set of all vectors $\left(y_{1}, \ldots, y_{n}\right)$ $\in \mathbb{R}^{n}$ satisfying

$$
\begin{aligned}
& y_{i} \geq 0, \quad 1 \leq i \leq n, \quad \text { and } \\
& y_{i}+y_{i+1} \leq 1, \quad 1 \leq i \leq n-1
\end{aligned}
$$

It follows from Corollary 4.2 that the volume $V_{n}$ of the set (9) satisfies

$$
\sum_{n \geq 0} V_{n} x^{n}=\sec x+\tan x
$$

Equation (10) was first given in [10] (see also [4]).

With almost no effort we obtain the following interesting corollary of Theorem 4.1.

Corollary 4.4. The order polynomial $\Omega(P, m)$ of a finite poset $P$ depends only on the comparability graph $\operatorname{Com}(P)$ of $P$.

Proof. By Theorem 4.1 we have $\Omega(P, m+1)=i(\mathscr{C}(P), m)$, and by definition $\mathscr{C}(P)$ depends only on $\operatorname{Com}(P)$.

In particular, the leading coefficient of $\Omega(P, m)$ depends only on $\operatorname{Com}(P)$, and we obtain

Corollary 4.5. The number $e(P)$ of linear extension of $P$ depends only on $\operatorname{Com}(P)$.

Corollary 4.5 was first stated in [7, p. 139]. Its proof was based on a condition as to when $\operatorname{Com}(P)=\operatorname{Com}(Q)$. This condition appears to be implicit in the work of Gallai and others, but was apparently first explicitly stated in [5], and is given as follows: Suppose $P$ contains a poset $P^{\prime}$ such that for all $x \in P-P^{\prime}$, either (a) $x<y$ for all $y \in P^{\prime}$, (b) $x>y$ for all $y \in P^{\prime}$, or (c) $x$ and $y$ are incomparable for all $y \in P^{\prime}$. Define $P_{1}$ to be the poset obtained from $P$ by dualizing $P^{\prime}$; i.e., $x \leq y$ in $P_{1}$ if and only if either (a) not both $x \in P^{\prime}$ and $y \in P^{\prime}$, and $x \leq y$ in $P$, or (b) $x$ and $y \in P^{\prime}$ and $x \geq y$ in $P$. Call $P_{1}$ a simple transform of $P$. Then $\operatorname{Com}(P)=$ $\operatorname{Com}(Q)$ if and only if there is a sequence $P=P_{0}, P_{1}, \ldots, P_{k}=Q$ of posets such that each $P_{i+1}$ is a simple transform of $P_{i}$. It is then easy to check that simple transforms have the same number of linear extensions, so Corollary 4.5 follows. In fact, it is just as easy to check that simple transforms have the same order polynomials, so Corollary 4.4 also follows. For another proof of Corollary 4.5 and additional references, see [8].

Note that the proof we gave of Corollary 4.4 really has nothing to do with convex polytopes. To see this, define for $m \geq 1$ the chain polynomial $\Gamma(P, m)$ to be the number of maps $g: P \rightarrow\{0,1,2, \ldots\}$ such that $g\left(y_{1}\right)+\cdots+g\left(y_{k}\right) \leq m-1$ for all chains $y_{1}<\cdots<y_{k}$ of $P$. Then (7) defines a bijection between order-preserving maps $f: P \rightarrow\{0, \ldots, m-1\}$ and maps $\phi f: P \rightarrow\{0,1, \ldots\}$ enumerated by $\Gamma(P, m)$. Hence $\Omega(P, m)=\Gamma(P, m)$. But $\Gamma(P, m)$ depends only on $\operatorname{Com}(P)$, so the same is true for $\Omega(P, m)$. 
Of course Corollary 4.4 may be extended to the statement that any invariant of $P$ which can be computed in terms of $\mathscr{C}(P)$ depends only on $\operatorname{Com}(P)$. In Corollary 6.3 we will see another example of such an invariant.

Corollary 4.4 and its proof suggest that the combinatorial type of $\mathcal{O}(P)$ itself may depend only on $\operatorname{Com}(P)$. However, if $P$ is given by Fig. 3 then there is easily seen to be a poset $Q$ satisfying $\operatorname{Com}(P)=\operatorname{Com}(Q)$ such that $O(Q)$ has nine facets, while $O(P)$ has eight facets.

\section{Triangulations}

The polytope $\mathcal{O}(P)$ has a canonical triangulation which can be transferred to $\mathscr{C}(P)$. We describe this procedure in this section and give an application in the next.

An order ideal of $P$ is a subset $I$ of $P$ such that if $x \in I$ and $y \leq x$, then $y \in I$. Let $J(P)$ denote the poset (actually a distributive lattice) of order ideals of $P$, ordered by inclusion. Let

$$
K: I_{1} \subset I_{2} \subset \cdots \subset I_{k}
$$

be a chain in $J(P)$ (where $I_{i-1} \subset I_{i}$ means that $I_{i-1}$ is strictly contained in $I_{i}$ ). Define a set $F_{K} \in \mathbb{R}^{P}$ by

$$
\begin{aligned}
F_{K}=\left\{f \in \mathbb{R}^{P} \text { : (a) } f\right. \text { is constant on the subsets } \\
\\
I_{1}, I_{2}-I_{1}, \ldots, I_{k}-I_{k-1}, P-I_{k} \text { of } P \text {, and } \\
\text { (b) } \left.0=f\left(I_{1}\right) \leq f\left(I_{2}-I_{1}\right) \leq \cdots \leq f\left(P-I_{k}\right)=1\right\} .
\end{aligned}
$$

Then $F_{K}$ is a $(k-1)$-dimensional simplex contained in $\mathcal{O}(P)$, and the set $\left\{F_{K}: K\right.$ is a chain of $J(P)\}$ is a triangulation $\Lambda(P)$ of $\mathscr{O}(P)$. [The empty chain $K$ corresponds to the empty face of $\Lambda(P)$.] In particular, the facets (maximal faces) of $\Lambda(P)$ are given by

$$
0 \leq f\left(y_{1}\right) \leq \cdots \leq f\left(y_{n}\right) \leq 1,
$$

where $y_{1}, \ldots, y_{n}$ is a linear extension of $P$. The number of facets is $e(P)$ and each has volume $1 / n$ !, giving another proof that $V(O(P))=e(P) / n !$.

For any poset $Q$ define the order complex $\Delta(Q)$ [1, Section 3] to be the abstract simplicial complex on $Q$ whose faces are the chains of $Q$. Hence, as an abstract simplicial complex, $\Lambda(P)$ is isomorphic to $\Delta(J(P))$. In particular, the geometric realization $|\Delta(J(P))|$ of $\Delta(J(P))$ is an $n$-cell, a result which also follows from very general considerations [11, Corollary 3.4.3] but here is explained more concretely.

It follows from the definition (7) of the transfer map $\phi$ that $\phi$ is linear on each face $F_{K}$ of $\Lambda(P)$. Hence $\phi\left(F_{K}\right)$ is a simplex, and (since $\phi$ is continuous) the set $\left\{\phi\left(F_{K}\right): F_{K} \in \Lambda(P)\right\}$ is a triangulation $\phi \Gamma(P)$ of $\mathscr{C}(P)$. By applying $\phi$ to the facet (11) of $\Lambda(P)$, an explicit description of the facets of $\phi \Lambda(P)$ can be deduced. Namely, given a linear extension $\sigma: P \rightarrow\{1, \ldots, n\}$ with $\sigma\left(y_{i}\right)=i$ and given 


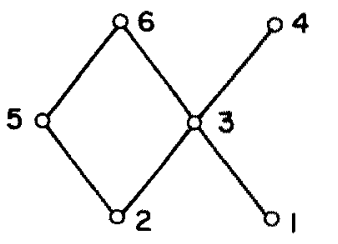

Fig. 4

$1 \leq k \leq n$, define a chain

$$
K_{y_{k}}=K_{k}: z_{j}<z_{j-1}<\cdots<z_{0}=y_{k}
$$

inductively by the conditions that (a) $z_{0}=y_{k}$; (b) among all $z$ covered by $z_{l}, \sigma(z)$ is maximized when $z=z_{i+1}$; and (c) $z_{j}$ is a minimal element of $P$. Let $F_{\sigma}$ be the facet (11) of $\Lambda(P)$; i.e., $F_{o}=F_{K}$, where $K$ is the maximal chain $\phi \subset\left\{y_{1}\right\} \subset$ $\left\{y_{1}, y_{2}\right\} \subset \cdots \subset P$ of $J(P)$. Then the equations defining the facet $\phi\left(F_{\sigma}\right)$ of $\phi \Lambda(P)$ are given by

$$
\begin{aligned}
0 & \leq f\left(y_{1}\right), \\
\sum_{x \in K_{1}} f(x) & \leq \sum_{x \in K_{t+1}} f(x), \quad 1 \leq i \leq n-1, \\
\sum_{x \in K_{n}} f(x) & \leq 1
\end{aligned}
$$

For instance, let $P$ and $\sigma$ be given by Fig. 4, where the element $y_{1}$ of $P$ is labeled $i$. Then, writing $f_{i}$ for $f\left(y_{i}\right)$, the equations for $\phi\left(F_{\sigma}\right)$ are given by

$$
\begin{aligned}
0 \leq f_{1} \leq f_{2} \leq f_{2}+f_{3} & \leq f_{2}+f_{3}+f_{4} \leq f_{2}+f_{5} \\
& \leq f_{2}+f_{5}+f_{6} \leq 1,
\end{aligned}
$$

which may also be written as

$$
\begin{aligned}
0 & \leq f_{1} \leq f_{2}, \quad 0 \leq f_{3}, \quad 0 \leq f_{4}, \quad 0 \leq f_{6}, \\
f_{3}+f_{4} & \leq f_{5}, \quad f_{2}+f_{5}+f_{6} \leq 1 .
\end{aligned}
$$

\section{Mixed Volumes}

In [16] the Alexandrov-Fenchel inequalities from the theory of mixed volumes were used to prove the logarithmic concavity of certain integer sequences associated with $\mathcal{O}(P)$. After reviewing this result we "transfer" it to $\mathscr{C}(P)$ and obtain new log-concave sequences involving linear extensions of $P$.

We state the Alexandrov-Fenchel inequalities in a form most convenient for our purposes. For references to their proofs, see [16]. Let $\left\{H_{\lambda}: 0 \leq \lambda \leq 1\right\}$ be a collection of parallel (affine) hyperplanes in $\mathbb{R}^{n}$ such that the distance between $H_{\lambda}$ and $H_{\mu}$ is $|\lambda-\mu|$. Let $\mathscr{P}_{0} \subset H_{0}$ and $\mathscr{P}_{1} \subset H_{1}$ be convex bodies (i.e., 
nonempty compact convex sets), and let $\mathscr{P}=\operatorname{cx}\left(\mathscr{P}_{0} \cup \mathscr{P}_{1}\right)$, the convex hull of $\mathscr{P}_{0}$ and $\mathscr{P}_{1}$. Set $\mathscr{P}_{\lambda}=\mathscr{P} \cap H_{\lambda}$ and let $V^{n-1}\left(\mathscr{P}_{\lambda}\right)$ denote the $(n-1)$-dimensional volume of $\mathscr{P}_{\lambda}$. Then there exist real numbers $V_{i}\left(\mathscr{P}_{0}, \mathscr{P}_{1}\right) \geq 0,0 \leq i \leq n-1$, such that

$$
V^{n-1}\left(\mathscr{P}_{\lambda}\right)=\sum_{i=0}^{n-1}\left(\begin{array}{c}
n-1 \\
i
\end{array}\right) V_{i}\left(\mathscr{P}_{0}, \mathscr{P}_{1}\right) \lambda^{i}(1-\lambda)^{n-1-i}, \quad 0 \leq \lambda \leq 1
$$

The number $V_{i}=V_{i}\left(\mathscr{P}_{0}, \mathscr{P}_{1}\right)$ is called the $i$ th mixed volume of $\mathscr{P}_{0}$ and $\mathscr{P}_{1}$ [in particular, $\left.\quad V_{0}\left(\mathscr{P}_{0}, \mathscr{P}_{1}\right)=V^{n-1}\left(\mathscr{P}_{0}\right), \quad V_{n-1}\left(\mathscr{P}_{0}, \mathscr{P}_{1}\right)=V^{n-1}\left(\mathscr{P}_{1}\right)\right], \quad$ and the Alexandrov-Fenchel inequalities assert that

$$
V_{i}^{2} \geq V_{i-1} V_{i+1}, \quad 1 \leq i \leq n-2
$$

Now consider the case $\mathscr{P}=\mathscr{O}(P)$, the order polytope of the $n$-element poset $P$. Fix $x \in P$, and for $0 \leq \lambda \leq 1$ set

$$
\mathcal{O}_{\lambda}=\{f \in \mathcal{O}(P): f(x)=\lambda\} \text {. }
$$

Then the $\mathcal{O}_{\lambda}$ s satisfy the conditions for (13). Moreover, if $\sigma$ is a linear extension of $P$ and $F_{\alpha}$ the corresponding facet (11) of $\Lambda(P)$ with $x=y_{1}$, then $\mathcal{O}_{\lambda} \cap F_{\sigma}$ is given by all $f \in \mathbb{R}^{P}$ satisfying

$$
0 \leq f\left(y_{1}\right) \leq \cdots \leq f\left(y_{i}\right)=\lambda \leq f\left(y_{i+1}\right) \leq \cdots \leq f\left(y_{n}\right) \leq 1 .
$$

It follows that

$$
V^{n-1}\left(\mathcal{O}_{\lambda} \cap F_{\alpha}\right)=\frac{\lambda^{i-1}(1-\lambda)^{n-i}}{(i-1) !(n-i) !}
$$

and hence

$$
V^{n-1}\left(\mathcal{O}_{\lambda}\right)=\frac{1}{(n-1) !} \sum_{i=0}^{n-1} N_{t+1}\left(\begin{array}{c}
n-1 \\
i
\end{array}\right) \lambda^{i}(1-\lambda)^{n-1-i},
$$

where $N_{j}$ is the number of linear extensions $\sigma$ of $P$ satisfying $\sigma(x)=j$. Therefore

$$
N_{t+1}=(n-1) ! V_{i}\left(\mathcal{O}_{0}, \mathcal{O}_{1}\right) \text {, }
$$

and we conclude from (13) that $N_{i}^{2} \geq N_{i-1} N_{i+1}, 2 \leq i \leq n-1$. More details are given in [16, Section 3] in a somewhat more general setting.

We now wish to "transfer" (15) and (16) to the chain polytope $\mathscr{C}=\mathscr{C}(P)$. We cannot simply define $\mathscr{C}_{\lambda}=\phi \mathcal{O}_{\lambda}$, since $\phi \mathcal{O}_{\lambda}$ need not lie in a hyperplane. Rather, we define $\mathscr{C}_{\lambda}$ in analogy to our definition of $\mathscr{O}_{\lambda}$ and compute $V\left(\mathscr{C}_{\lambda}\right)$ by examining each $\psi \mathscr{C}_{\lambda} \cap F_{\sigma}$, where $\psi$ is given by (8). Thus fix $x \in P$, and for $0 \leq \lambda \leq 1$ set

$$
\mathscr{C}_{\lambda}=\{f \in \mathscr{C}(P): f(x)=\lambda\}
$$


Lemma 6.1. $\mathscr{C}(P)=\operatorname{cx}\left(\mathscr{C}_{0} \cup \mathscr{C}_{1}\right)$

Proof. By Theorem 2.2 every vertex of $\mathscr{C}(P)$ lies in $\mathscr{C}_{0} \cup \mathscr{C}_{1}$, and the proof follows.

It follows that there are numbers $M_{0}, M_{1}, \ldots, M_{n-1}$ (depending on the choice of $x \in P$ ) uniquely defined by

$$
(n-1) ! V^{n-1}\left(\mathscr{C}_{\lambda}\right)=\sum_{i=0}^{n-1} M_{i}\left(\begin{array}{c}
n-1 \\
i
\end{array}\right) \lambda^{i}(1-\lambda)^{n-1-1}, \quad 0 \leq \lambda \leq 1
$$

and that then $M_{i}^{2} \geq M_{i-1} M_{i+1}, 1 \leq i \leq n-2$. It remains to interpret $M_{i}$ combinatorially.

Theorem 6.2. $M_{i}$ is equal to the number of linear extensions $\sigma: P \rightarrow\{1, \ldots, n\}$ such that if $\sigma(x)=s$, then $i$ is the largest integer (necessarily less than $s$ ) for which $\sigma^{-1}(s-1), \sigma^{-1}(s-2), \ldots, \sigma^{-1}(s-i)$ are all incomparable with $x$. (In particular, $i=0$ if $s=1$ or if $\sigma^{-1}(s-1)<\sigma^{-1}(s)$ in $P$. If $x$ is a minimal element of $P$ then $i=s-1$.)

Proof. Since the simplices $\phi F_{\sigma}$ are the facets of the triangulation $\phi \Lambda(P)$ of $\mathscr{C}(P)$, we have

$$
V^{n-1}\left(\mathscr{C}_{\lambda}\right)=\sum_{\sigma} V^{n-1}\left(\mathscr{C}_{\lambda} \cap \phi F_{\sigma}\right)
$$

summed over all linear extensions $\sigma$ of $P$. Define a map $p: \mathbb{R}^{P} \rightarrow \mathbb{R}^{P-\{x\}}$ by restricting $f \in \mathbb{R}^{P}$ to $P-\{x\}$. Since $p$ is a projection orthogonal to $\mathscr{C}_{\lambda}$, we have $V^{n-1}\left(\mathscr{C}_{\lambda} \cap \phi F_{\mathrm{o}}\right)=V\left(p\left(\mathscr{C}_{\lambda} \cap \phi F_{\sigma}\right)\right)$, where $V$ denotes ordinary $(n-1)$-dimensional volume (Lebesgue measure) in $\mathbb{R}^{P-\{x\}} \cong \mathbb{R}^{n-1}$. Let $\psi: \mathscr{C}(P) \rightarrow \mathscr{O}(P)$ be the bijection defined by (8). Consider the composition $p \psi: \mathscr{C}_{\lambda} \rightarrow \mathbb{R}^{P-\{x\}}$. From (8) it follows that for any $y \in P-\{x\}$ and any $g \in \mathscr{C}_{\lambda} \cap \phi F_{\sigma}$, we have

$$
(p \psi G)(y)=\sum_{z \in K_{y}} g(z)
$$

where $K_{y}$ is the chain (12) defined in Section 5 (and where $g(x)=\lambda$ by the definition of $\mathscr{C}_{\lambda}$ ). Hence the map $p \psi$, when restricted to $\mathscr{C}_{\lambda} \cap \phi F_{\sigma}$, is an affine transformation whose linear part can be put in triangular form with 1 s on the diagonal. In particular, $p \psi$ is volume-preserving, so

$$
V^{n-1}\left(p \psi\left(\mathscr{C}_{\lambda} \cap \phi F_{\sigma}\right)\right)=V^{n-1}\left(p\left(\psi \mathscr{C}_{\lambda} \cap F_{\alpha}\right)\right)=V^{n-1}\left(\mathscr{C}_{\lambda} \cap \phi F_{\sigma}\right)
$$

The first equality holds because $\psi \phi=$ identity.

Let $y$ be that element of $P$ covered by $x$ which maximizes $\sigma(y)$. Then the condition $g(x)=\lambda$ for $g \in \mathscr{C}_{\lambda} \cap \phi F_{0}$ is by (7) equivalent to $f(x)-f(y)=\lambda$ in $\psi\left(\mathscr{C}_{\lambda} \cap \phi F_{\sigma}\right)$. [If $x$ is minimal then the condition becomes $f(x)=\lambda$.] Define $y_{i}=\sigma^{-1}(i)$ and suppose $y=y_{r}, x=x_{s}$. Set $f_{i}=f\left(y_{i}\right)$. Then the set $p \psi\left(\mathscr{C}_{\lambda} \cap \phi F_{\sigma}\right)$ 
is defined by the conditions

$$
0 \leq f_{1} \leq \cdots \leq f_{s-1} \leq f_{r}+\lambda \leq f_{s+1} \leq \cdots \leq f_{n} \leq 1
$$

For fixed $f_{r}=t$ (where $0 \leq t \leq 1-\lambda$ ) the projection of (20) orthogonal to the plane $f_{r}=t$ has $(n-2)$-dimensional volume

$$
\frac{t^{r-1}}{(r-1) !} \frac{\lambda^{s-r-1}}{(s-r-1) !} \frac{(1-\lambda-t)^{n-s}}{(n-s) !} .
$$

Hence

$$
\begin{aligned}
V^{n-1}\left(p \psi\left(\mathscr{C}_{\lambda} \cap \phi F_{\mathrm{o}}\right)\right)= & \frac{\lambda^{s-r-1}}{(r-1) !(s-r-1) !(n-s) !} \\
& \times \int_{0}^{1-\lambda} t^{r-1}(1-\lambda-t)^{n-s} d t
\end{aligned}
$$

Let $t=u(1-\lambda)$ to obtain

$$
\int_{0}^{1-\lambda} t^{r-1}(1-\lambda-t)^{n-s} d t=(1-\lambda)^{n+r-s} \int_{0}^{1} u^{r-1}(1-u)^{n-s} d u
$$

This latter integral is just the beta function

$$
B(r, n-s+1)=\frac{(r-1) !(n-s) !}{(n+r-s) !}
$$

Hence

$$
V^{n-1}\left(p \psi\left(\mathscr{C}_{\lambda} \cap \phi F_{\sigma}\right)\right)=\frac{\lambda^{s-r-1}(1-\lambda)^{n+r-s}}{(s-r-1) !(n+r-s) !}
$$

Set $s-r-1=h(\sigma)=h(\sigma, x)$. Comparing (18), (19), and (21) yields

$$
(n-1) ! V^{n-1}\left(\mathscr{C}_{\lambda}\right)=\sum_{\sigma}\left(\begin{array}{l}
n-1 \\
h(\sigma)
\end{array}\right) \lambda^{h(\sigma)}(1-\lambda)^{n-1-h(\sigma)}
$$

But clearly $h(\sigma)$ is just the largest integer $i$ for which $\sigma^{-1}(s-1), \sigma^{-1}(s-$ $2), \ldots, \sigma^{-1}(s-i)$ are all incomparable with $x$, and the proof follows by comparing (17) and (22).

Example 6.3. Let $P$ be given by Fig. 5, with $x_{i}$ labeled $i$. Choose $x=x_{4}$. We list the linear extensions of $P$, with the elements $\sigma^{-1}(s-1), \ldots, \sigma^{-1}(s-i)$ incom- 
<smiles>O[Mg]O[Mg]</smiles>

parable with $x$ underlined:

$12 \underline{3} 45$

$21 \frac{1}{3} 45$

12435

21435

24135

12453

21453

24153

24513

Hence $M_{0}=4, M_{1}=3, M_{2}=1, M_{3}=M_{4}=0$.

Since $\mathscr{C}(P)$ depends only on $\operatorname{Com}(P)$ we obtain, just as for Corollary 4.4 , the following corollary.

Corollary 6.4. For any n-element poset $P$ and any $x \in P$, the numbers $M_{0}, M_{1}, \ldots, M_{n-1}$ defined in Theorem 6.2 depend only on $\operatorname{Com}(P)$ with the vertex $x$ specified.

It is not even a priori obvious that the $M_{i}$ s are unaffected by replacing $P$ with its dual $P^{*}$ (and leaving the choice of $x$ unaltered), but a simple combinatorial proof which we omit can be given. More generally, Corollary 6.4 can also be proved using the result of Gallai et al. discussed after Corollary 4.5 .

Just as Theorem 6.2 is the " $\mathscr{C}(P)$ analogue" of (16), so Theorem 6.2 and its consequence $M_{i}^{2} \geq M_{i-1} M_{i+1}$ can be straightforwardly generalized to give a $\mathscr{C}(P)$ analogue of the generalization of (16) given in [16, Thm. 3.2]. Moreover, a variation of (15) given in $[9,(2.14)]$ can also be given a $\mathscr{C}(P)$ analogue. We will not enter into details here.

A general property of the mixed volumes $V_{i}$ of (13) asserts that if $V_{i}=0$ then either $V_{0}=V_{1}=\cdots=V_{i}=0$ or $V_{i}=V_{i+1}=\cdots=V_{n-1}=0$. This property, together with (14) and the fact that $V_{i} \geq 0$, implies that the sequence $V_{0}, V_{1}, \ldots, V_{n-1}$ is unimodal; i.e., for some $j$ we have $V_{0} \leq V_{1} \leq \cdots \leq V_{j} \geq V_{j+1} \geq \cdots \geq V_{n-1}$. In the case of the numbers $M_{i}$ of Theorem 6.2, an even stronger result can be proved by direct combinatorial reasoning.

Theorem 6.5. The numbers $M_{i}=M_{i}(x)$ of Theorem 6.2 are weakly decreasing; i.e., $M_{0} \geq M_{1} \geq \cdots \geq M_{n-1}$. 
Proof. Let $\Omega_{i}$ be the set of linear extensions $\sigma$ of $P$ satisfying $h(\sigma)=i$, where $h(\sigma)$ is as in (22). If $i>0$ and $\sigma \in \Omega_{i}$, then define $\sigma^{\prime}: P \rightarrow\{1, \ldots, n\}$ by

$$
\sigma^{\prime}(y)= \begin{cases}\sigma(y), & \text { if } y \neq x \text { and } \sigma(y) \neq \sigma(x)-1 \\ \sigma(x)-1, & \text { if } y=x \\ \sigma(x), & \text { if } \sigma(y)=\sigma(x)-1 .\end{cases}
$$

The map $\sigma \mapsto \sigma^{\prime}$ is an injection from $\Omega_{i}$ into $\Omega_{i-1}$. Since $\left|\Omega_{r}\right|=M_{i}$, the proof follows.

\section{References}

1. A. Björner, A. Garsia, and R. Stanley, An introduction to the theory of Cohen-Macaulay partially ordered sets, in Ordered Sets (I. Rival, ed.), Reidel, Dordrecht-Boston-London, 1982, $583-616$.

2. V. Chvátal, On certain polytopes associated with graphs, J. Combin. Theory Ser B 18 (1975), $138-154$.

3. L. Comtet, Advanced Combinatorics, Reidel, Dordrecht-Boston, 1974.

4. E. E. Doberkat, Problem 84-20, SIAM Review 26 (1984), 580.

5. B. Dreesen, W. Poguntke, and P. Winkler, Comparability invariance of the fixed point property, preprint.

6. L. Geissinger, A polytope associated to a finite ordered set, preprint.

7. M. C. Golumbic, Algorithmic Graph Theory and Perfect Graphs, Academic Press, New York, 1980.

8. M. Habib, Comparability invariants, Ann. Discrete Math. 23 (1984), 371-386.

9. J. Kahn and M. Saks, Balancing poset extensions, Order 1 (1984), 113-126.

10. I. G. Macdonald and R. B. Nelsen (independently), Solution to E2701, Amer. Math. Monthly 86 (1979), 396.

11. J. S. Provan and L. J. Billera, Simplicial Complexes Associated with Convex Polyhedra, I: Constructions and Combinatorial Examples, Technical Rept. no. 402, School of Operations Research and Industrial Engineering, Cornell University, Ithaca, New York, January 1979.

12. R. Stanley, A chromatic-like polynomial for ordered sets., in Proc. Second Chapel Hill conference on Combinatorial Mathematics and Its Applications (May, 1970). Univ. of North Carolina, Chapel Hill, 421-427.

13. R. Stanley, Ordered structures and partitions, Mem. Amer. Math. Society, no. 119, 1972.

14. R. Stanley, Eulerian partitions of a unit hypercube, in Higher Combinatorics (M. Aigner, ed.), Reidel, Dordrecht-Boston, 1977, p. 49.

15. R. Stanley, Decompositions of rational convex polytopes, Ann. Discrete Math. 6 (1980), $333-342$.

16. R. Stanley, Two combinatorial applications of the Aleksandrov-Fenchel inequalities, J. Combin. Theory Ser A 31 (1981), 56-65.

Received February 19, 1985. 